\title{
ON CONJUGACY OF SUPPLEMENTS OF NORMAL SUBGROUPS OF FINITE GROUPS
}

\author{
A. BALLESTER-BOLINCHES ${ }^{\bowtie}$ and LUIS M. EZQUERRO \\ (Received 19 March 2013; accepted 15 April 2013; first published online 13 June 2013)
}

\begin{abstract}
The objective of this paper is to find some sufficient conditions to ensure the conjugacy of supplements of a normal subgroup of a soluble group.
\end{abstract}

2010 Mathematics subject classification: primary 20D10; secondary 20D20.

Keywords and phrases: finite groups, supplements, Schunck classes, formations.

\section{Introduction}

All groups considered in this paper are finite. We shall adhere to the notation and terminology of $[1,3]$.

We recall that a formation is a class of groups $\mathfrak{F}$ which is closed under taking epimorphic images and subdirect products. We say that $\mathfrak{F}$ is saturated if a group $G$ belongs to $\mathfrak{F}$ whenever the Frattini factor group $G / \Phi(G)$ belongs to $\mathfrak{F}$. A Schunck class is a class of groups such that a group is in the class if and only if every primitive factor group is in the class. It is known that a formation is a Schunck class if and only if it is saturated.

Our main goal here is to find some sufficient conditions to ensure the conjugacy of supplements of a normal subgroup of a soluble group. Our results spring basically from two sources: classical results about projectors associated to Schunck classes and saturated formations; and a recent result of Parker and Rowley on conjugacy of supplements of a nilpotent normal subgroup [4].

The search for conjugacy classes of subgroups to give knowledge on the structure of the groups, has attracted the attention of many group theorists after the celebrated Sylow theorems (see [1, 3]). In this context, in [4] the authors proved the following result.

The reseach of the authors is supported by Proyecto MTM2010-19938-C03-01/03 of the Ministerio de Ciencia e Innovación de España. The first author is also supported by Project of NSFC (11271085).

(c) 2013 Australian Mathematical Publishing Association Inc. 0004-9727/2013 \$16.00 
THEOREM 1.1. Let $G$ be a soluble group and $Q$ a nilpotent normal subgroup of $G$ such that no $G$-chief factor of $G / Q$ is $G$-isomorphic to a $G$-chief factor of $Q$. If $U$ and $V$ are supplements to $Q$ in $G$ with $U \cap Q=V \cap Q$, then $U$ and $V$ are $G$-conjugate.

This theorem can be easily interpreted in terms of Schunck classes and projectors and can be deduced as a consequence of a known result of this theory. In fact, we can consider the Schunck class $\mathfrak{H}$ of all soluble groups whose primitive epimorphic images belong to the class of all primitive epimorphic images of $G / Q$, and we can argue by minimal counterexample. Then $G=\left\langle U, V^{g}\right\rangle$, for all $g \in G$, and $U \cap Q=V \cap Q=1$. A well-known lemma on projectors due to Gaschütz [3, III, 3.14] implies that $U$ and $V$ are contained in $\mathfrak{h}$-projectors $U^{*}$ and $V^{*}$ of $G$ respectively. Hence $G \in \mathfrak{H}$, a contradiction proving the result.

The question arises whether it is possible to find sufficient conditions on $Q$, weaker than nilpotency, and sufficient conditions on the intersections $U \cap Q$ and $V \cap Q$, weaker than equality, to imply the conjugacy of $U$ and $V$. The results of this paper give us some answers.

\section{Supplemented normal subgroups}

If we turn the situation on its head and look for structural conditions on a normal subgroup $Q$ of a not necessarily soluble group $G$ having a conjugacy class of supplements, we have the following theorem.

Theorem 2.1. Suppose that $G$ is a group and $Q$ is a normal subgroup of $G$ such that any two maximal subgroups of $G$ supplementing $Q$ in $G$ are $G$-conjugate. Then $Q$ is a soluble group of nilpotent length at most 2 .

Proof. We suppose that the theorem is false and derive a contradiction. Let $(G, Q)$ be a counterexample with $G$ of minimal order. Let $N$ be a nontrivial normal subgroup of $G$ and consider the quotient group $\bar{G}=G / N$. Write $\bar{Q}=Q N / N$. Suppose that $\bar{U}=U / N$ and $\bar{V}=V / N$ are two maximal subgroups of $\bar{G}$ supplementing $\bar{Q}$ in $\bar{G}$. Then $U$ and $V$ are two maximal subgroups of $G$ which are supplements to $Q$ in $G$. By hypothesis $U$ and $V$ are $G$-conjugate. Therefore $\bar{U}$ and $\bar{V}$ are $\bar{G}$-conjugate. It follows from the minimal choice of $G$ that $\bar{Q}$ is a group of nilpotent length at most 2. Since the class of group of nilpotent length at most 2 is a saturated formation, it follows that $G$ has a unique minimal normal subgroup, $N$ say, and $N \leq Q$.

If $N \leq \Phi(G)$, then $Q$ is of nilpotent length at most 2, by [2, Theorem 3.5]. Therefore $\Phi(G)=1$. This means that $\operatorname{Soc}(G)=N$ is a supplemented minimal normal subgroup of $G$.

Suppose that $N$ is nonsoluble. Then $G$ is a primitive group of type 2 whose core-free maximal subgroups are $G$-conjugate. This is not possible (see [1, Ch. 1, Section 1.1, Paragraph 1.1.11, Remark 4]). Hence $N=\operatorname{Soc}(G)$ is an abelian minimal normal subgroup of $G$.

Let $U$ be a core-free maximal subgroup of $G$. Then $U$ is a complement to $N$ in $G$. Write $R=U \cap Q$ and $A=\mathrm{F}(R)$. Then $Q=R N$. Since $R \cong Q / N$ has nilpotent length 
at most 2, we have that $R / A$ is nilpotent. Note that $R$ is a normal subgroup of $U$ and so is $A$. If $A \leq \Phi(U)$, then $R$ would be nilpotent, by [2, Theorem 3.5], and $Q$ would be a group of nilpotent length at most 2. But then $G$ would not be a counterexample. Therefore $A$ is not contained in $\Phi(U)$.

Let $S$ be a maximal subgroup of $U$ such that $U=A S$ and consider the subgroup $V=S N$. Then $V$ is maximal in $G$ and $G=U N=A S N=A V=Q V$. In other words, $V$ is a supplement to $Q$ in $G$. By hypothesis, $V$ and $U$ are conjugate. This is not possible because $N \leq V$ and $N \cap U=1$. Therefore no counterexample exists and $Q$ is of nilpotent length at most 2 .

The bound of Theorem 2.1 is best possible, as the following example shows.

Example 2.2. Consider the group $X=\operatorname{SL}(2,3)$ acting on a two-dimensional vector space $V$ over the Galois field $\mathrm{GF}(3)$. Construct the semidirect product $G=[V] X$. Recall that $X=P C$, where $P$ is a normal subgroup of $X$ and $P$ is isomorphic to the quaternion group of order eight and $C$ is a cyclic group of order three. If $Z=Z(X)$, the centre of $X$, then $Q=Z V$ is a supersoluble nonnilpotent normal subgroup of $G$. The set of maximal subgroups supplementing $Q$ in $G$ is the conjugacy class of all core-free maximal subgroups of $G$ complementing $V$.

\section{The converse}

Next we present some results which can be viewed as partial converses of Theorem 2.1. In fact, our second theorem describes a minimal configuration encountered in the study of conjugacy of supplements of normal nilpotent subgroups of soluble groups, from which sufficient conditions and counterexamples emerge. The following example shows that some conditions should be imposed on the intersections.

ExAmple 3.1. Let $G=\left\langle a, b, x: a^{3}=b^{3}=x^{2}=1=[a, b], a^{x}=a^{-1}, b^{x}=b^{-1}\right\rangle$. If $Q=$ $\langle a, b\rangle \cong C_{3} \times C_{3}$, then the subgroups $U=\langle a, x\rangle$ and $V=\langle b, x\rangle$ are two supplements to $Q$ in $G$ which are not $G$-conjugate.

Note that in this example, $U \cap Q=\langle a\rangle$ and $V \cap Q=\langle b\rangle$ are two different normal subgroups of $G$, which are not $G$-conjugate.

It seems reasonable, therefore, to impose local conjugacy on the intersections since a minimal configuration usually implies that $Q$ is of prime power order.

Recall that two subgroups $A$ and $B$ of a group $G$ are locally $G$-conjugate if every Sylow subgroup of $A$ is $G$-conjugate to a Sylow subgroup of $B$ (see [3, Ch. I, Section 6, Definitions (6.13)]). Local conjugacy is an important subgroup embedding property in soluble groups.

Theorem 3.2. Let $\mathcal{X}$ be a $\mathrm{Q}$-closed class of groups and let $\mathfrak{N}$ be the class of nilpotent groups. Consider the class

$$
\mathcal{F}=\mathfrak{N} \mathcal{X}=(G: G / M \in \mathcal{X} \text { for some nilpotent normal subgroup } M \text { of } G) .
$$


Let $G$ be a soluble group of minimal order in $\mathcal{F}$ among the groups satisfying the following property:

$(\dagger)$ there exists a nilpotent normal subgroup $Q$ of $G$ and non-G-conjugate supplements $U$ and $V$ of $Q$ in $G$ such that $U \cap Q$ is locally $G$-conjugate to $V \cap Q$.

Then $G$ is a p-group for some prime $p$.

Proof. Let $Q$ be of minimal order among all nilpotent normal subgroups of $G$ supplemented by two non- $G$-conjugate subgroups $U$ and $V$ in $G$ such that $U \cap Q$ is locally $G$-conjugate to $V \cap Q$. Then $1 \neq Q$ and $U$ and $V$ are proper subgroups of $G$.

The proof is divided into five steps, the first being a standard argument in proofs involving conjugation.

Step 1. Let $N$ be a minimal normal subgroup of $G$ contained in $Q$. Then $V^{g} N=U N$ for some $g \in G$.

Consider the quotient group $\bar{G}=G / N$. Then $\bar{Q}=Q / N$ is a nilpotent normal subgroup of $\bar{G}$ and $\bar{G} / \bar{Q} \in \mathcal{F}$. Write $\bar{U}=U N / N$ and $\bar{V}=V N / N$. Then $\bar{U}$ and $\bar{V}$ are supplements to $\bar{Q}$ in $\bar{G}$. Moreover, $\bar{U} \cap \bar{Q}=(U \cap Q) N / N$ and $\bar{V} \cap \bar{Q}=(V \cap Q) N / N$. Therefore $\bar{U} \cap \bar{Q}$ and $\bar{V} \cap \bar{Q}$ are locally $\bar{G}$-conjugate. The minimal choice of $G$ implies that $V^{g} N=U N$ for some element $g \in G$.

Step 2. $Q$ is a p-group for some prime $p$.

If $p$ is a prime dividing the order of $Q$, let $Q_{p}$ denote the Sylow $p$-subgroup of $Q$. If $Q_{p} \leq U$, then $Q_{p}$ is the Sylow $p$-subgroup of $U \cap Q$ and since $Q_{p}$ is normal in $G$, we have that $Q_{p} \leq V$ by hypothesis. Therefore there exists a prime $p$ dividing the order of $Q$ such that $U$ is a proper subgroup of $U Q_{p}$. Since $Q_{p}$ is normal in $G$, there exists an element $g \in G$ such that $U Q_{p}=V^{g} Q_{p}$, by Step 1. Denote $X=U Q_{p}=V^{g} Q_{p}$. Note that $U \cap Q_{p}$ is a Sylow $p$-subgroup of $U \cap Q$ and $V^{g} \cap Q_{p}$ is a Sylow $p$-subgroup of $V^{g} \cap Q$. Since $G=X Q=X Q_{p^{\prime}}$, where $Q_{p^{\prime}}$ is the Hall $p^{\prime}$-subgroup of $Q$, it follows that $U \cap Q_{p}$ and $V^{g} \cap Q_{p}$ are $X$-conjugate. Moreover, $X /(X \cap Q) \cong G / Q$. Therefore $X \in \mathcal{F}$. If $X$ is a proper subgroup of $G$, then $U$ and $V^{g}$ are $G$-conjugate and this is not possible. Hence $G=X$. Now if $Q_{p}$ is a proper subgroup of $Q$, then $U$ and $V^{g}$ are conjugate by minimality of $Q$. Therefore $Q$ is a $p$-group.

Step 3.

(1) $G=\left\langle U, V^{x}\right\rangle$ for any $x \in G$; and

(2) $Q$ is a minimal normal subgroup of $G$.

By Step 2, there exists an element $g \in G$ such that $Q \cap U=(Q \cap V)^{g}=Q \cap V^{g}$. Consider the subgroup $Z=\left\langle U, V^{g}\right\rangle$ and assume that $Z$ is a proper subgroup of $G$. Then $Z=U Q \cap Z=U(Q \cap Z)=V^{g}(Q \cap Z)$. Moreover, $G / Q \cong Z /(Z \cap Q)$, since $G=Z Q$, and then $Z \in \mathcal{F}$. Now

$$
U \cap Q \cap Z=U \cap Q=V^{g} \cap Q=V^{g} \cap Q \cap Z .
$$

By minimality of $G$, the subgroups $U$ and $V^{g}$ are $Z$-conjugate. Hence $U$ and $V$ are $G$-conjugate, contrary to supposition. Therefore $G=Z=\left\langle U, V^{g}\right\rangle$. This implies 
that $U \cap Q=V^{g} \cap Q$ is normalised by $U$ and $V^{g}$. Thus $U \cap Q$ is normal in $G$ and $U \cap Q=V \cap Q$. Now, if $x \in G$, then $U \cap Q=V^{x} \cap Q$. Arguing as above, $G=\left\langle U, V^{x}\right\rangle$.

Let $N$ be a minimal normal subgroup of $G$ contained in $Q$. Then, by Step 1 , $U N=V^{x} N$ for some $x \in G$. Then $G=U N=V^{x} N$. If $U=G$, we have $V \leq G$ and $Q=Q \cap U$. Since $Q$ is a nilpotent normal subgroup of $G$, we have $Q \leq V$ and then $G=V$. Then $U$ and $V$ are trivially $G$-conjugate. Hence $U$ and $V^{x}$ are maximal subgroups of $G$ complementing $N$. Then $Q=N$ by the minimal choice of $G$.

Step 4. $\mathrm{O}_{p^{\prime}}(U)=1$.

Suppose that $\mathrm{O}_{p^{\prime}}(U) \neq 1$ and let $M$ be a minimal normal $p^{\prime}$-subgroup of $U$. Then $Q=[M, Q] \times \mathrm{C}_{Q}(M)$ by [3, Ch. A, Section 12, Theorem (12.5)]. Note that $U$ normalises $[M, Q]$ and $\mathrm{C}_{Q}(M)$. Thus they are normal subgroups of $G$. By minimality of $Q$, we have that either $\mathrm{C}_{Q}(M)=1$ or $\mathrm{C}_{Q}(M)=Q$.

Suppose that $\mathrm{C}_{Q}(M)=Q$. Then $M$ is normal in $G$. This implies that $M \leq \mathrm{O}_{p^{\prime}}(G)$. Since $V$ contains a Hall $p^{\prime}$-subgroup of $G$, we have that $M \leq U \cap V$. By Step $1, U$ and $V$ are $G$-conjugate. Therefore $\mathrm{C}_{Q}(M)=1$. Hence $Q$ is a $\mathbb{Z} M$-module whose $M$ submodule $Q^{M}$ composed of all fixed points is trivial. In particular, $\mathbf{H}^{1}\left(U / M, Q^{M}\right)=0$. By [3, Ch. A, Section 11, Theorem (11.3)] $), \mathbf{H}^{1}(M, Q)=0$. Applying [5, Ch. III, Section 3.4, Proposition 3.4.2], the sequence

$$
0 \longrightarrow \mathbf{H}^{1}\left(U / M, \mathrm{C}_{Q}(M)\right)=0 \stackrel{\mathrm{inf}}{\longrightarrow} \mathbf{H}^{1}(U, Q) \stackrel{\text { res }}{\longrightarrow} \mathbf{H}^{1}(M, Q)=0
$$

is exact. This implies that $\mathbf{H}^{1}(U, Q)=0$. This is equivalent to saying that all complements of $Q$ in $G$ are conjugate. In particular, $U$ and $V$ are $G$-conjugate. This contradiction yields $\mathrm{O}_{p^{\prime}}(U)=1$.

Step 5. Conclusion.

By Step 4, we have that $\mathrm{O}_{p}(U) \neq 1$ and $\mathrm{O}_{p}(G)=Q \mathrm{O}_{p}(U)=Q \mathrm{O}_{p}(V)$. Assume that $\mathrm{O}_{p}(G)$ is a proper subgroup of $G$. The minimal choice of $G$ implies that $\mathrm{O}_{p}(U)=\mathrm{O}_{p}(V)^{g}$ for some $g \in \mathrm{O}_{p}(G)$. Then $\mathrm{O}_{p}(U)$ is normalised by $U$ and by $V^{g}$ and then $\mathrm{O}_{p}(U)$ is normal in $G=\left\langle U, V^{g}\right\rangle$. In particular, $\mathrm{O}_{p}(U) \leq U \cap V$. By step $1, U$ and $V$ are $G$-conjugate, contrary to supposition. Hence $G$ is a $p$-group.

Theorem 3.2 allows us to obtain a number of results on conjugacy of supplements of nilpotent normal subgroups of soluble groups.

If $\mathcal{Y}$ is a class of soluble primitive groups then by [3, Ch. III, Section 2, Theorem (2.7)] the class $\mathfrak{H} y$ composed of all soluble groups whose primitive epimorphic images are in $\mathcal{Y}$ is a Schunck class.

Corollary 3.3. Let $G$ be a soluble group and $Q$ a nilpotent normal subgroup of $G$ such that no $G$-chieffactor of $G / Q$ is $G$-isomorphic to a $G$-chieffactor of $Q$.

If $U$ and $V$ are supplements to $Q$ in $G$ such that $U \cap Q$ and $V \cap Q$ are locally $G$ conjugate, then $U$ and $V$ are $G$-conjugate.

Proof. Let $\mathcal{Y}$ be the class of all primitive groups which are isomorphic to a factor group of $G / Q$. Consider the Schunck class $\mathfrak{H}=\mathfrak{H} y$. Then $G / Q \in \mathfrak{H}$ and then $G \in \mathfrak{N} \mathfrak{H}$. 
If $G$ is a minimal counterexample to the corollary, then $G$ is a group of minimal order in $\mathfrak{N H}$ satisfying $(\dagger)$. By Theorem 3.2, $G$ is a $p$-group. Therefore $\mathfrak{H}=\mathfrak{\Xi}_{p}$, the class of $p$-groups. But then the hypothesis forces $Q=G$ or $Q=1$. If $Q=1$, then $U=V=G$. If $G=Q$, then $U$ and $V$ are $G$-conjugate by hypothesis. Thus no possibility can happen and the minimal counterexample does not exist.

Note that Theorem 1.1 is a particular case of Corollary 3.3.

We shall show now by an example that no statement of a similar kind is possible if we remove the hypothesis on the chief factors.

ExAmple 3.4. Let $Q$ be a group isomorphic to the quaternion group of order eight. Then $\operatorname{Aut}(Q)$ is isomorphic to $S$, the symmetric group on four letters. Consider a subgroup $T$ of $S$ isomorphic to the symmetric group on three letters. Write $T=\left\langle b, c: b^{3}=c^{2}=1, b^{c}=b^{-1}\right\rangle$. Set $B=\langle b\rangle$ and $C=\langle c\rangle$. Construct the semidirect product $G=[Q] T$. Write $Z=\mathrm{Z}(Q)=\langle z\rangle$. Note that $G / Q B$ is a complemented central 2-chief factor of $G$ over $Q$ and $Z / 1$ is also a central $G$-chief factor of $G$. Consider the subgroups $U=\langle b, c\rangle$ and $V=\langle b, z c\rangle$. Then $U$ and $V$ are two supplements to $Q$ in $G$. Moreover, $U \cap Q=1=V \cap Q$.

If $U^{g}=V$ for some $g \in G$, then $g \in \mathrm{N}_{G}(\langle b\rangle)=Z \times U$. This would imply that $U=V$. But then $z=(z c) c \in U$ and this is not true.

Corollary 3.5. Suppose that $G$ is a soluble group and $Q$ is a nilpotent normal subgroup of $G$. If $U$ and $V$ are supplements to $Q$ in $G$ such that $U$ and $V$ are locally $G$-conjugate, then $U$ and $V$ are $G$-conjugate.

Proof. Consider the class $\mathcal{X}$ of all soluble groups $X$ such that $X$ possesses a nilpotent normal subgroup $W$ supplemented in $X$ by two locally $X$-conjugate proper subgroups $Y$ and $Z$ of $X$. Clearly the class $\mathcal{X}$ is Q-closed. Write $\mathcal{F}=\mathfrak{N} X$.

Let $G$ be a minimal counterexample to the corollary. There exists a nilpotent normal subgroup $Q$ of $G$ and supplemented by two non- $G$-conjugate subgroups $U$ and $V$ which are locally $G$-conjugate. Hence $U \cap Q$ and $V \cap Q$ are locally $G$-conjugate. Therefore $G$ is a group of minimal order in $\mathcal{F}$ among the groups satisfying $(\dagger)$. By Theorem 3.2, $G$ is a $p$-group for some prime $p$. Then $U$ and $V$ are $G$-conjugate. This is the desired contradiction.

Let $\mathfrak{F}$ be a saturated formation. If $G$ is a group and $G \notin \mathfrak{F}$, then the $\mathfrak{F}$-residual $G^{\mathfrak{F}}$ of $G$, the smallest normal subgroup of $G$ with quotient in $\mathfrak{F}$, is a nontrivial normal subgroup of $G$ which is supplemented in $G$ by every $\mathfrak{F}$-projector of $G$.

Corollary 3.6. Let $\mathfrak{F}$ be a saturated formation and let $G$ be a soluble group whose $\mathfrak{\Im}$-residual $G^{\widetilde{\mho}}$ is nilpotent. Then any two supplements $U$ and $V$ of $G^{\mathscr{\mho}}$ in $G$ are $G$-conjugate provided $U \cap G^{\widetilde{F}}$ and $V \cap G^{\widetilde{F}}$ are locally $G$-conjugate.

Proof. Let $\mathcal{F}$ be the class of all groups whose $\mathfrak{F}$-residual is nilpotent. Then $\mathcal{F}=\mathfrak{N} F$. If $G$ is minimal counterexample to the corollary, then $G$ is a group of minimal order in $\mathfrak{N} \mathfrak{F}$ satisfying $(\dagger)$. Applying Theorem 3.2 to the class $\mathcal{F}$, it follows that $G$ is a $p$-group. 
Hence either $G^{\mathscr{F}}=G$, and then $U$ and $V$ are $G$-conjugate by hypothesis, or $G^{\mathscr{F}}=1$, and then $U=V=G$. In any case $G$ is not a counterexample and the corollary holds.

The case where $G^{\mathscr{\mho}}$ is abelian is particularly interesting. In this case $G^{\mathfrak{夭}}$ is complemented in $G$ and its complements form a conjugacy class of subgroups of $G$ by [3, Ch. IV, Section 5, Theorem (5.18)]. We see that when $G^{\widetilde{r}}$ is abelian, Corollary 3.6 is equivalent to the fact that the complements of $G^{\tilde{F}}$ are conjugate in $G$.

Corollary 3.7. Let $\mathfrak{F}$ be a saturated formation and let $G$ be a soluble group whose $\mathfrak{F}$-residual $G^{\mathfrak{夭}}$ is abelian. The following conditions are equivalent:

(1) any two supplements $U$ and $V$ of $G^{\mathfrak{F}}$ in $G$ are $G$-conjugate provided $U \cap G^{\mathscr{S}}$ and $V \cap G^{\widetilde{F}}$ are locally $G$-conjugate;

(2) any two complements $U$ and $V$ of $G^{\mathscr{F}}$ in $G$ are $G$-conjugate.

Proof. Since clearly (1) $\Rightarrow(2)$ we have only to prove $(2) \Rightarrow(1)$. Assume that the F-residual $G^{\mathfrak{F}}$ is abelian and let $U$ and $V$ be two supplements of $G^{\mathscr{\mho}}$ in $G$ with $U \cap G^{\mathfrak{\preccurlyeq}}$ and $V \cap G^{\mathfrak{r}}$ locally $G$-conjugate. If $P$ is a Sylow $p$-subgroup of $U \cap G^{\mathscr{\mho}}$ and $R$ is a Sylow $p$-subgroup of $V \cap G^{\widetilde{\mho}}$, then $R=P^{x}$ for some $x \in U$. Therefore $R \leq U \cap G^{\widetilde{\mho}}$ and then $R=P$. Therefore $U \cap G^{\mathscr{f}}=V \cap G^{\mathscr{f}}$. Moreover $N=U \cap G^{\tilde{F}}$ is normal in $G$. Then $U / N$ and $V / N$ are complements of $G^{\mathscr{F}} / N=(G / N)^{\tilde{F}}$ in $G / N$. By (2), $U$ and $V$ are conjugate in $G$.

\section{References}

[1] A. Ballester-Bolinches and L. M. Ezquerro, Classes of Finite Groups (Springer, Dordrecht, 2006).

[2] A. Ballester-Bolinches and M. D. Pérez-Ramos, 'On ₹ $₹$-subnormal subgroups and Frattini-like subgroups of a finite group', Glasgow Math. J. 36 (1994), 241-247.

[3] K. Doerk and T. Hawkes, Finite Soluble Groups (Walter de Gruyter, Berlin, 1992).

[4] C. Parker and P. Rowley, 'A note on conjugacy of supplements in finite soluble groups', Bull. Lond. Math. Soc. 42 (2010), 417-419.

[5] E. Weiss, Cohomology of Groups (Academic Press, New York, 1969).

\section{A. BALLESTER-BOLINCHES, Departament d'Àlgebra, Universitat de València, Dr. Moliner, 50, E-46100 Burjassot, València, Spain e-mail: Adolfo.Ballester@uv.es}

LUIS M. EZQUERRO, Departamento of Matemáticas, Universidad Pública de Navarra, Campus de Arro-sadía, E-31006 Pamplona, Navarra, Spain e-mail: ezquerro@unavarra.es 